\title{
BINDING OF BIEXCITONS IN GaAs/AlGaAs SUPERLATTICES
}

\author{
V. MizeIKIS \\ Institute of Material Research and Applied Science, Vilnius University \\ Saulétekio Ave. 9, build. III, 2054 Vilnius, Lithuania \\ D. Birkedal, W. Langbein, V.G. Lyssenko and J.M. Hvam \\ Mikroelektronik Centret, The Technical University of Denmark \\ 2800 Lyngby, Denmark
}

Binding of the heavy-hole excitons and biexcitons in $\mathrm{GaAs} / \mathrm{Al}_{0.3} \mathrm{Ga}_{0.7} \mathrm{As}$ superlattices is studied using linear and nonlinear optical techniques. High biexciton binding energies characteristic of quasi two-dimensional biexcitons are observed in superlattices with considerable miniband dispersion.

PACS numbers: 71.35.Cc, 78.66.Hf, 42.50.Md

Binding energies of Coulomb-bound states, namely the heavy-hole (HH) excitons and biexcitons in GaAs/AlGaAs multiple quantum wells (MQW) and superlattices (SL) are significantly enhanced compared to bulk GaAs. The main reason for this enhancement is the one-dimensional confinement of the electrons and holes along the direction of growth. By now it is established that in the limiting three-dimensional (3D) and two-dimensional (2D) cases, the ratio of the biexciton to the exciton binding energy $\sigma=E_{x x}^{\mathrm{b}} / E_{x}^{\mathrm{b}}$ is a constant. In 3D it is given by the Haynes rule [1]:

$$
\sigma \approx 0.1
$$

while in $2 \mathrm{D}$ [2]:

$$
\sigma \approx 0.228 \text {. }
$$

It is interesting to investigate this ratio for intermediately confined systems which are neither strictly 2D nor 3D. Obviously, a transformation from (2) to (1) must accompany the 2D-3D transition. In MQW systems, where the confinement is mostly determined by the geometric GaAs well thickness $L_{\mathrm{w}}$, a constant value of $\sigma \approx 0.2$ in agreement to (2) was found, while $L_{\mathrm{w}}$ was varied within the $80-160 \AA$ range [3].

In this work we present a similar study of GaAs/AlGaAs samples having the same well width, but different AlGaAs barrier widths $L_{\mathrm{b}}$. Thus, we investigate the transition from a MQW to SL structure, and associated change of dimensionality. The samples investigated are MBE-grown on (100) semi-insulating GaAs substrates, and consist of $20 \mathrm{GaAs} / \mathrm{Al}_{0.3} \mathrm{Ga}_{0.7}$ As periods. In all samples 
$L_{\mathrm{w}}=80 \AA$, while $L_{\mathrm{b}}=200,100,50,30$ and $20 \AA$. In the following we refer to the samples by their barrier thickness. All experiments were carried out at a temperature of $4 \mathrm{~K}$. The exciton binding energy was obtained from linear optical absorption spectra and calculations, while the biexciton binding energy was measured from the exciton-biexciton quantum beats in transient four-wave mixing (TFWM) experiments [4]. TFWM experiments are performed using femtosecond pulses (duration $120 \mathrm{fs}$ FWHM) from a $76 \mathrm{MHz}$ repetition rate Ti:sapphire laser. The two-beam self-diffraction geometry [5] with time-integrated signal detection was used. The TFWM signal was recorded as a function of the delay $t_{12}$ between two coherent laser pulses. For a three-level system, coherently excited by a spectrally broad laser pulse, the signal intensity $I_{\text {TFWM }}$ decays exponentially, and contains harmonic quantum beat modulations of the form $\cos \left[(\Delta E / \hbar) t_{12}\right]$, where $\Delta E$ is the difference between the energies of two excited states. Thus, from the beat period $\Delta E$ can be determined. To obtain maximum information in the TFWM experiment [6], the signal is spectrally-resolved, and recorded using the optical multichannel analyzer system.

The exciton binding energy $E_{x}^{\mathrm{b}}$ can be determined from the linear absorption spectrum as the difference between the $1 s$ absorption peak and the onset of the band-to-band continuum transitions according to Elliott formula [7]. With resonant excitation, biexcitons can be created via two-photon transitions with a single exciton as an intermediate state [8]. The biexciton binding energy $E_{x x}^{\mathrm{b}}$ in this case can be determined from the energy difference between the biexciton and exciton resonance enhancements in the TFWM spectrum, or in delay domain, from the period of the exciton-biexciton quantum beats [4].

The linear absorption spectra of samples are shown in Fig. 1. For better comparison they are shifted by an amount $\Delta$ along the energy axis in order to make the

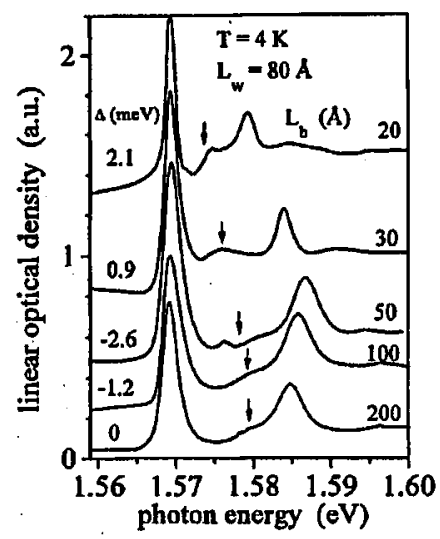

Fig. 1. Spectra of linear optical density in GaAs/AlGaAs quantum wells and superlattices. The onset of the $\mathrm{HH}$ continuum transitions, corresponding to the calculated values of $E_{x}^{\mathrm{b}}$ (see Table), is marked by the arrows. $\Delta$ indicates the horizontal offset for each spectrum. 
HH exciton peaks in all plots coincident. The onset of HH continuum transitions is seen as a broad shoulder of $8-12 \mathrm{meV}$ above the HH exciton resonance. The absolute energies of the heavy-hole and light-hole (LH) excitonic peaks vary slightly from sample to sample, most likely due to accidental mechanical strains occurring when thin samples are mounted on the sapphire substrates. These random shifts are compensated by the horizontal offsets in Fig. 1. The above circumstances make it difficult to determine accurately $E_{x}^{\mathrm{b}}$ from the linear absorption spectra. Despite this, Fig. 1 reveals clear signatures of the superlattice formation with decreasing barrier width. In the 200 and $100 \AA$ samples, which consist of almost perfectly isolated quantum wells, $E_{x}^{\mathrm{b}} \approx 11 \mathrm{meV}$ can be inferred from Fig. 1 . For $L_{\mathrm{b}} \leq 50 \AA, E_{x}^{\mathrm{b}}$ decreases to near 6 and $5 \mathrm{meV}$ in the 30 and $20 \AA$ samples, respectively. The latter value is already close to that in bulk GaAs at $T=4 \mathrm{~K}$. The HH-LH splitting, which is a direct consequence of the carrier confinement, decreases for $L_{\mathrm{b}} \leq 50 \AA$.

For further demonstration of the fact that the spectra in Fig. 1 show superlattice formation, we have calculated the exciton binding energies using the fractional-dimensional approach [9] (see [10] for more details). Parameters of GaAs, required for the calculations, were taken from literature [11], and $k \cdot p$ band-structure calculations. Some results relevant for this work are given in Table. As seen from the table, electron and heavy-hole minibands become broader, and accordingly, carriers delocalize along the superlattice growth direction with decrease in the barrier thickness. This trend is more pronounced for the electrons, which have smaller in-plane effective mass, while the heavy-holes remain substantially localized. The arrows in Fig. 1 indicate the onset of the HH continuum transitions, corresponding to the calculated exciton binding energies. The experimental and theoretical data in the figure are in reasonable agreement.

\section{TABLE}

Calculated electron and hole miniband widths $\Delta E_{e}$ and $\Delta E_{\mathrm{hh}}$, combined miniband halfwidth $\Delta E / 2$, and $\mathrm{HH}$ exciton binding energy in GaAs/AlGaAs superlattices.

\begin{tabular}{c|c|c|c|c}
\hline \hline$L_{\mathrm{b}}[\AA]$ & $\Delta E_{\mathrm{e}}[\mathrm{meV}]$ & $\Delta E_{\mathrm{hh}}[\mathrm{meV}]$ & $\Delta E / 2[\mathrm{meV}]$ & $E_{x}^{\mathrm{b}}[\mathrm{meV}]$ \\
\hline 200 & $\rightarrow 0$ & $\rightarrow 0$ & $\rightarrow 0$ & 9.2 \\
100 & 0.14 & $\rightarrow 0$ & 0.07 & 9.2 \\
50 & 3.3 & $\rightarrow 0$ & 1.65 & 7.7 \\
30 & 12 & 0.8 & 6.4 & 6.0 \\
20 & 23.5 & 2.3 & 12.9 & 5.2
\end{tabular}

Figure 2 shows the TFWM signal, measured about $2.5 \mathrm{meV}$ below the energy of the $\mathrm{HH}$ exciton. The pronounced oscillations, seen for all the samples, except the one with $L_{\mathrm{b}}=20 \AA$, are the exciton-biexciton quantum beats. Their period $\approx 1.6 \mathrm{ps}$ yields $E_{x x}^{\mathrm{b}} \approx 2.6 \mathrm{meV}$ in all samples, which exhibit the beating. In the $20 \AA$ sample no beating was detected below the HH exciton energy, most likely due to very low biexciton binding energy in this sample. Supposedly, at a certain critical barrier thickness $20 \AA<L_{\mathrm{b}}<30 \AA$ biexcitons loose their quasi-two-dimensional character and spread along the superlattice growth direction with accompanying 


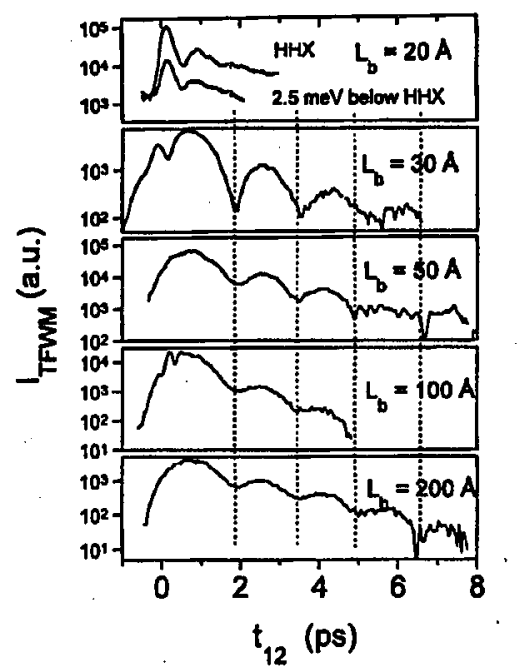

Fig. 2. Decays of the TFWM signal as a function of delay $t_{12}$ at the spectral position of the heavy-hole biexciton in samples with different barrier width. Vertical dashed lines are guides to the eye marking the signal minima. In the top plot, the signal decays in the $20 \AA$ sample, measured at the $\mathrm{HH}$ exciton energy (a) and $2.5 \mathrm{meV}$ below it (b) are shown.

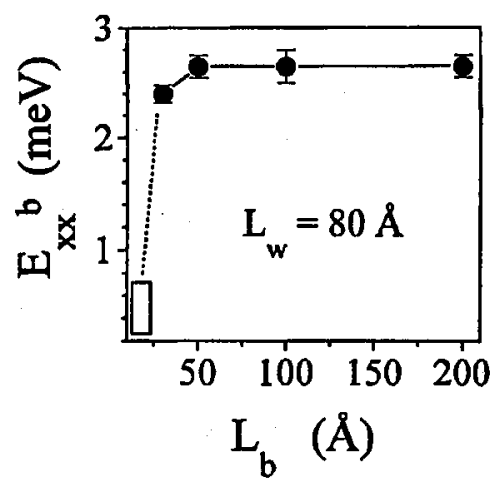

Fig. 3. Heavy-hole biexciton binding energy as a function of barrier width. The dotted line and $\square$ show the expected behavior of the biexciton binding energy due to the $2 \mathrm{D}$-bulk transition, and its value in the $20 \AA$ sample.

decrease in the binding energy. For bulk biexcitons the 3D Ilaynes rule (1) applies, and due to their low binding energy $(\leq 0.5 \mathrm{meV})$ these states may become unstable even at helium temperature. Even if a biexcitonic contribution to the TFWM signal would remain, the quantum beat period would exceed the $\mathrm{HH}$ exciton dephasing time, and thus become impossible to determine. Figure 3 shows that in the $30 \AA$ sample $E_{x x}^{\mathrm{b}}$ is slightly smaller than in the 50,100 , and $200 \AA$ samples, indicating possible onset of the abrupt change. In the figure we also include the tentative 
value of $E_{x x}^{\mathrm{b}} \approx 0.5 \mathrm{meV}$ for $L_{\mathrm{b}}=20 \AA$. Hence, $\sigma$ increases with barrier width going from 30 to $200 \AA$. For the 200 and $100 \AA$ samples we obtain $\sigma \approx 0.24$ in agreement with earlier findings in MQW systems [3]. For the 50 and $30 \AA$ samples $\sigma=0.34$ and 0.43 , respectively, is inferred. Finally, for the $20 \AA$ sample we expect sharp drop in $\sigma$ as the 3D Haynes rule (1) becomes effective.

Thus, our most important observation is that the biexcitonic states retain their quasi two-dimensionality and high binding energy of $2.6 \mathrm{meV}$, as $L_{\mathrm{b}}$ decreases from 200 to $30 \AA$. The excitons, in contrast, seem to loose their quasi two-dimensional character, as is evidenced by their decreasing binding energy. Strong biexciton binding in 200 and $100 \AA$ samples is not surprising, since in these samples adjacent quantum wells are almost completely isolated from each other by thick barriers. In these structures excitons, biexcitons, and free carriers are confined within a single GaAs well. But in the 50 and $30 \AA$ samples, where the interwell interaction is considerable, we have an openly conflicting situation, where a localized biexciton is formed by the interaction between two delocalized excitons.

This can be solved by realizing, that in fact, the excitons in the latter two samples are localized just like the biexcitons. It is appropriate at this point to recall that so called narrow-miniband superlattices satisfy the relation [12]

$$
\Delta E / 2<E_{x}^{\mathrm{b}, \mathrm{MQW}},
$$

where $\Delta E$ is the combined width of the electron and HH minibands, and $E_{x}^{\text {b,MQW }}$ is the exciton binding energy in noninteracting quantum wells of the same thickness as in the superlattice. Relation (3) compares two opposite trends, namely the electron delocalization in a periodic superlattice potential, and localization by the Coulomb potential of the heavy hole. Choosing the appropriate value of $E_{x}^{\mathrm{b}, \mathrm{MQW}} \approx 11 \mathrm{meV}$ found in the $200 \AA$ sample, we see from Table, that all the investigated samples except the one with $L_{\mathrm{b}}=20 \AA$ fall into the category of narrow miniband superlattices. It was shown earlier $[12,13]$ that in narrow miniband GaAs superlattices, the heavy-hole Coulomb potential effectively reduces electron penetration into the adjacent GaAs wells. Thus, resulting $\mathrm{HH}$ exciton states are predominantly localized within a single superlattice period. This circumstance is responsible for the suppression of Stark-ladder transitions in biased superlattices [12], and formation of the interwell exciton [3]. The same arguments can be applied for the biexcitons, since their formation lowers the electron energy even more. In practice this situation corresponds to all samples except the one with $L_{\mathrm{b}}=20 \AA$. As the superlattice barrier width decreases from 30 to $20 \AA$, condition (3) is violated, and the hole potential cannot localize the electrons anymore. Both excitons and biexcitons will spread in space over several superlattice periods, acquiring three-dimensional degrees of freedom. This assumption is also supported by the fact that in the $20 \AA$ sample the $\mathrm{HH}$ exciton line width $(\approx 1.5 \mathrm{meV}$. FWHM) is somewhat smaller than in the other samples $(\approx 2.2 \mathrm{meV})$. This indicates that only in the $20 \AA$ sample the excitons indeed delocalize, spreading over several GaAs/AlGaAs periods, and becoming less sensitive to local well-width and alloy fluctuations, which are the main source of inhomogeneous broadening in MBE-grown MQW structures. 
The abrupt nature of the transition from the two-dimensional to the three-dimensional behavior can be explained as follows. It was shown earlier that in $2 \mathrm{D}$ it is possible to transform the Hamiltonian of a biexciton into that of a single exciton with modified parameters [2]. The validity of this model for the $80-160 \AA$ quantum wells has been proven experimentally [3]. In treating the biexcitons as excitons it is relevant to refer to variational calculations by Chomette et al. [14] showing that even though the decrease in the exciton binding energy is fairly smooth, the exciton radius in the growth direction increases abruptly when the superlattice period $L_{\mathrm{b}}+L_{\mathrm{w}}$ reaches a critical value. In our case of biexcitons it corresponds to that, at a critical barrier thickness (in the $20<L_{\mathrm{b}}<30 \AA$ range), the two-dimensional model of biexciton breaks down and we observe an abrupt transition to the bulk state.

In conclusion, we have studied the biexciton binding in GaAs/AlGaAs superlattices with different barrier thicknesses and miniband widths. We have observed that the $\mathrm{HH}$ biexcitons preserve their quasi $2 \mathrm{D}$ character despite substantial superlattice miniband dispersion. Transition to bulk character occurs in a step-like manner at certain critical barrier thickness. These features are explained in terms of the localization, mediated by the Coulomb interaction in the narrow miniband superlattices.

This work was supported by the Danish Ministries of Industry and Research in the framework of CNAST. All the samples used were grown by C.B. Sørensen.

\section{References}

[1] J. Haynes, Phys. Rev. Lett. 17, 860 (1966).

[2] J. Singh, D. Birkedal, V. Lyssenko, J. Hvam, Phys. Rev. B 53, 15909 (1996).

[3] D. Birkedal, J. Singh, V.G. Lyssenko, J. Erland, J.M. Hvam, Phys. Rev. Lett. 76, 672 (1996).

[4] K.-H. Pantke, D. Oberhauser, V.G. Lyssenko, J.M. Hvam, G. Weimann, Phys. Rev. $B$ 47, 2413 (1993).

[5] L. Schultheis, M.D. Sturge, J. Hegarty, Appl. Phys. Lett. 47, 995 (1985).

[6] J. Erland, K.-H. Pantke, V. Mizeikis, V.G. Lyssenko, J.M. Hvam, Phys. Rev. B 50, 15047 (1994).

[7] R.J. Elliott, Phys. Rev. 108, 1384 (1957).

[8] D. Lovering, R. Philips, G. Denton, G. Smith, Phys. Rev. Lett. 68, 1880 (1992).

[9] X.-F. He, Phys. Rev. B 43, 2063 (1991).

[10] V. Mizeikis, D. Birkedal, W. Langbein, V.G. Lyssenko, J.M. Hvam, Phys. Rev. B 55, 5284 (1997).

[11] Properties of Aluminium Gallium Arsenide, EMIS Datareviews No. 7, Ed. S. Adachi, INSPEC, London 1993.

[12] A.M. Fox, D.A.B. Miller, J.E. Cunningham, W.Y. Yan, C.Y.P. Chao, S.L. Chuang, Phys. Rev. B 46, 15365 (1992).

[13] G. Cohen, I. Bar-Joseph, H. Shtrikman, Phys. Rev. B 50, 17316 (1994).

[14] A. Chomette, B. Lambert, B. Devedaud, F. Clerot, A. Regreny, G. Bastard, Europhys. Lett. 4, 461 (1987). 